\title{
Identification of Mine-Shaped Objects based on an Efficient Phase Stepped-Frequency Radar Approach
}

Sørensen, Helge Bjarup Dissing; Jakobsen, Kaj Bjarne; Nymann, Ole

Published in:

Proceedings ICIP-97

Link to article, DOI:

10.1109/ICIP.1997.632027

Publication date:

1997

Document Version

Publisher's PDF, also known as Version of record

Link back to DTU Orbit

Citation $(A P A)$ :

Sørensen, H. B. D., Jakobsen, K. B., \& Nymann, O. (1997). Identification of Mine-Shaped Objects based on an Efficient Phase Stepped-Frequency Radar Approach. In Proceedings ICIP-97 (pp. 142-145). IEEE Computer Society Press. https://doi.org/10.1109/ICIP.1997.632027

\section{General rights}

Copyright and moral rights for the publications made accessible in the public portal are retained by the authors and/or other copyright owners and it is a condition of accessing publications that users recognise and abide by the legal requirements associated with these rights.

- Users may download and print one copy of any publication from the public portal for the purpose of private study or research.

- You may not further distribute the material or use it for any profit-making activity or commercial gain

- You may freely distribute the URL identifying the publication in the public portal 


\title{
Identification of Mine-Shaped Objects based on an Efficient Phase Stepped-Frequency Radar Approach
}

\author{
Helge B. D. Sorensen ${ }^{a}$, Kaj B. Jakobsen ${ }^{a}$, and Ole Nymann ${ }^{b}$ \\ aDepartment of Applied Electronics, Bldg. 451, Technical University of Denmark, DK-2800 Lyngby \\ ${ }^{b}$ Center for Advanced Technology, CAT Science Park, DK-4000 Roskilde, Denmark
}

\begin{abstract}
A computational efficient approach to identify very small mine-shaped plactic objects, e.g. M56 AntiPersonnel (AP) mines buried in the ground, is presented. The size of the objects equals the smallest AP-mines in use today, i.e., the most difficult mines to detect with respect to humanitarian mine clearance. Our approach consists of three stages, the phase stepped-frequency radar method, generation of a quarternary image and template crosscorrelation. The phase stepped-frequency radar method belongs to the class of stepped-frequency radar methods, see e.g [1]. In a two-dimensional meshgrid above the ground a radar probe is moved automatically to measure in each grid point a set of reflection coefficients from which phase and amplitude information are extracted. Based on a simple processing of the phase information, quarternary image and template cross-correlation a successfull detection of metal- and non-metal mine-shaped objects is possible. Measurements have been performed on loamy soil containing different mine-shaped objects.
\end{abstract}

\section{1: Introduction}

The detection of buried and non-buried objects have been examined using different types of radar. The most widely used is the video or pulse radar [3], the frequency modulated continuous wave (FM-CW) radar [4], interferometric radar [5], and stepped frequency radar [1], [2]. Our experiments are based on the stepped frequency radar approach.

H.B.D.S.: E-mail: hbs@iae.dtu.dk; WWW: http:/www.dtu.dk; Telephone: +45 45255244 ; Fax: +45 45880286. K.B.J.: E-mail: kbj@iae.dtu.dk; WWW: http://www.dtu.dk; Telephone: 4454525 5255 ; Fax: +45 4588 0286. O.N.: Emmail: cat-olny@cat.risoe.dk; Telephone: +45 46775919 ; Fax: +4546321919
Our experimental setup is illustrated in Fig. 1. The backbone in the measurement system is the linear bearings where the probe is mounted. The probe is an open waveguide operating at S-band. The probe is used both as transmitter and receiver, i.e., monostatic operation, to avoid using two probes. The probe can be moved in the $\mathrm{x}$ and $\mathrm{y}$ directions in increments down to 1 $\mathrm{mm}$. The movements of the probe are regulated by the stepmotors via an RS-232 interface to the PC. The probe is connected via a $5 \mathrm{~m}$ long Sucoflex cable to an HP8753C network analyzer that is set up to measure the amplitude and phase of the reflection coefficient. The calibrated network analyzer is connected to the PC via an HPIB interface for automated data collection. The collected data at each physical measurement point from a two-dimensional mesh-grid consist typically of reflection coefficient measurement at $N$ frequencies evenly spaced over a selected frequency range. Our approach for the identification of the mine-shaped objects is presented in the following section.

\section{2: The Approach}

The objective is humanitarian mine clearance by using identification of metallic- and non-metallic mines in the ground.

Six mine-shaped metal- and non-metallic objects have been buried in loamy soil. The center-coordinates of the mine-shaped objects in the soil are shown in Table 1. The $x$ - and $y$-coordinates indicate the object center position inside a rectangular surface area with the $x-y$ dimension $1410 \mathrm{~mm} \times 210 \mathrm{~mm}$. The z-position indicates the distance to the object in the soil. All the objects have the same irregular shape with a diameter of $54 \mathrm{~mm}$ and height of $40 \mathrm{~mm}$.

The stepped-frequency principle is realized by measuring the reflection coefficients $\Gamma(f, x, y)$ at different 


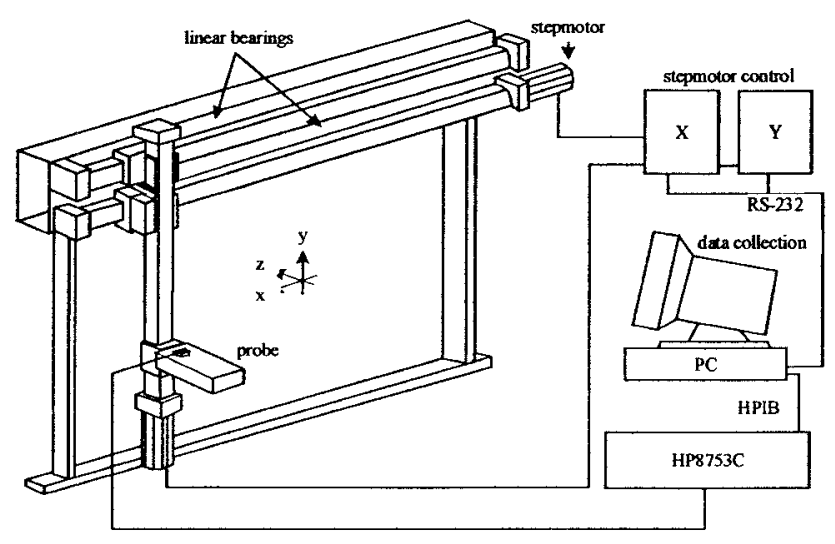

Fig. 1 Experimental setup. The position of the probe is controlled by the PC via the RS232 to the step motors.

frequencies $f$ for each position of the probe. For a fixed $y$ position of the probe, it is stepped $M$ times in the xdirection using a step size of e.g. $\Delta x=10 \mathrm{~mm}$.

Our approach for identification of mine-shaped objects buried in the ground is presented in the following subsections and it consists of three stages, the phase stepped-frequency radar method, generation of a quarternary image and template crosscorrelation.

The first step in our approach is the phase steppedfrequency radar method and this is based on the use of the following phase-based expression:

$$
\Gamma_{\angle}(x, y)=\int_{f_{L}}^{f_{H}} \angle \Gamma(f, x, y) d f
$$

where $\mathrm{f}_{\mathrm{L}}$ is the low and $\mathrm{f}_{\mathrm{H}}$ is the high frequency. We have observed that this kind of averaging improves the appearance of the mine objects in a radar image compared to a raw phase (radar) image. In practice we are using a discrete version of $\Gamma_{L}(\mathrm{x}, \mathrm{y})$ described by:

$$
\Gamma_{\angle}(x, y)=\sum_{i=N_{3}}^{N_{2}} \angle \Gamma\left(f_{\text {start }}+\left(i-N_{1}\right) \cdot \Delta f, x, y\right),
$$

where $f_{\text {start }}$ is the start frequency and $\Delta \mathrm{f}$ is the frequency step size. $N_{1}$ and $N_{2}$ are frequency indices (integers). The number of frequency indices is denoted $N=N_{2}-N_{1}+1$.

The second step in the approach is a kind of image enhancement realized by the generation of a quarternary image by quantizing the dynamic range in the image $\Gamma_{L}(\mathrm{x}, \mathrm{y})$ into four levels defined by:
TABLE 1

Center-coordinates

of the mine-shaped objects in the ground.

\begin{tabular}{|c|c|c|c|}
\hline Position & $x(\mathrm{~mm})$ & $y(\mathrm{~mm})$ & $z(\mathrm{~mm})$ \\
\hline Wooden & 0 & 100 & -30 \\
\hline Plastic & 230 & 100 & -30 \\
\hline M-56 & 460 & 100 & -30 \\
\hline Brass & 690 & 100 & -30 \\
\hline Aluminum & 920 & 100 & -30 \\
\hline Steel & 1150 & 100 & -30 \\
\hline
\end{tabular}

$$
\Gamma_{\angle}^{\prime}(x, y)=\left\{\begin{array}{lr}
\frac{3}{2}(\mu+\sigma), & \Gamma_{\angle}(x, y) \geq \mu+\sigma \\
\mu+\frac{\sigma}{2}, & \mu \leq \Gamma_{\angle}(x, y)<\mu+\sigma \\
\mu-\frac{\sigma}{2}, & \mu-\sigma \leq \Gamma_{\angle}(x, y)<\mu \\
\frac{1}{2}(\mu-\sigma), & \Gamma_{\angle}(x, y)<\mu-\sigma .
\end{array}\right.
$$

The defined data ranges are more or less natural ranges. The levels have been selected empirically.

The third step in our approach is the so-called template crosscorrelation. More complicated template matching methods based on e.g. dynamic time warping principles or Markov models could be applied but they are not necessarily desirable due to the computational load. The template crosscorrelation is calculated as:

$$
R_{\Gamma, M}(n, m)=\Gamma_{\measuredangle}^{\prime}(x+n-2, y+m-2) \star M(n, m),
$$

where $\star$ denotes crosscorrelation. $M(n, m)$ is a square matrix representing a binary mine template. A crosscorrelation between the mine template and each possible submatrix of similar size from $\Gamma^{\prime}{ }_{2}(x, y)$ can indicate/enhance positions of the mines. The following expression is used to generate a new image by selecting the center of $\mathrm{R}_{\Gamma, M}(n, m)$ for each template matching:

$$
\Gamma_{\angle}^{\prime \prime}(x, y)=R_{\Gamma, M}\left(\frac{n+1}{2}, \frac{m+1}{2}\right) .
$$

The main idea behind the definitions of all the expressions in this section is the desire of simple and efficient calculations due to the large amount of data in large radar images. Fast mine detection is very important in humanitarian mine clearance tasks. 


\section{3: Results}

Initially we have buried six mine-shaped metal- and non-metal objects in a box containing soil. The objects have the same shape as an M56 plastic AP-mine and the diameter and height is 54 and $40 \mathrm{~mm}$, respectively. The plastic/wax object is an M-56 AP-mine (dummy).

For a fixed $y$-position the probe is stepped $M$ times in the $\mathrm{x}$-direction using $\Delta \mathrm{x}=10 \mathrm{~mm}$ (alternatively 20 or $30 \mathrm{~mm}$ ) to generate, e.g., an amplitude- or a phasespectrogram extracted from the reflection coefficients. A spectrogram represents the frequency content along one physical scan line, i.e., the y-position of the probe is fixed. Fig. 2 and 3 show an amplitude- and phasespectrogram, fixed at $\mathrm{y}=100 \mathrm{~mm}$ and $\Delta \mathrm{x}=10 \mathrm{~mm}$.

Our phase stepped-frequency radar method was inspired by the facts that there are similarities between the amplitude- and phase-spectrograms with respect to the response on mine-shaped objects. We selected the phase-spectrograms as a starting point. More elaborated processing, which was beyond the scope of this work, will probably use both amplitude and phase information.

The phase stepped-frequency radar method is based on collapsing each phase-spectral line vector in a phasespectrogram matrix. In this way the matrix is collapsed into a vector. In our initial experiments we have defined collaps operation as a simple summation to reduce the computational load and to make noise reduction in the spectrogram possible due to the averaging.

Fig. 4 presents the initial results based on our method. All the subfigures in Fig. 4 and the following figures are based on measurements using a probe scanning a rectangular surface area with the $x-y$ dimensions $1,410 \mathrm{~mm} \times 210 \mathrm{~mm}$. In Fig. 4 (a), (b), (c) the resolutions are $\Delta x=\Delta y=30 \mathrm{~mm}, \Delta x=\Delta y=20 \mathrm{~mm}$, and $\Delta x=\Delta y=10 \mathrm{~mm}$, respectively. Fig. 4 (a), (b) and (c) are thus generated from 7,10 , and 21 collapsed phasespectrograms, respectively. The bandwidth of each spectrogram was set to the frequency range 3,600-3,900 $\mathrm{MHz}$ with a frequency step of $3 \mathrm{MHz}$, i.e., the number of frequency samples $N$ is 100 . Only the fundamental mode is present in the waveguide. All metal- and non-metal objects described in Table 1 can be visually identified in Fig. 4 (b) and (c) using our method except the wooden object, i.e., a harmless and irrelevant object. These results indicate that the probe has to be moved in steps $\leq 20 \mathrm{~mm}$.

Our phase stepped-frequency radar method has furthermore been tested with respect to the bandwidth. Fig. 5 illustrates the performance of the method as a function of the bandwidth. The frequency range used to generate phase-spectrograms in Fig. 5 are 3,825-3,900 $\mathrm{MHz}(N=25), 3,750-3,900 \mathrm{MHz}(N=50)$, and 3,600$3,900 \mathrm{MHz}(N=100)$ in Fig. 5 (a), (b) and (c), respectively. The frequency step size is $3 \mathrm{MHz}$ in all cases. The resolutions in Fig. 5 are set to $\Delta x=\Delta y=10$ $\mathrm{mm}$. From Fig. 5 it is observed that the bandwidth can be reduced without loss of relevant information. The efficiency of our method can be determined by using Fig. 4 (b) and (c). The computational load per $\mathrm{m}^{2}$ is $N$ phase extractions at $N$ frequencies at $2.4 \cdot 10^{3}-1.0 \cdot 10^{4}$ measurement points and $2.4 \cdot 10^{3}-1.0 \cdot 10^{4}$ summations. Our experiments show that $N$ should be between 25-100. The reported results are all based on experiments with mineshaped objects buried in soil. Experiments based on other ground materials may give different results.

Fig. 7 illustrates the advantage of using quarternary image generation compared to not using this as seen in Fig. 6. In Fig. 6 (c) we see the result of using the phase stepped-frequency radar method, followed by template crosscorrelation applying a binary mine template $5 \times 5$ matrix. In Fig. 7 (c) we see the result of using the three stage processing consisting of the phase steppedfrequency radar method, quarternary image generation and template crosscorrelation applying a binary mine template $(5 \times 5)$ matrix. The result in Fig. 7 (c) gives a better identification of the five mine objects compared to Fig. 6 (c). This result emphasizes the advantage of having a three stage processing.

\section{4: Conclusion}

A fast three stage processing consisting of the phase stepped-frequency radar method, quarternary image generation and template crosscorrelation applying a binary mine template matrix on radar images has shown that successful identification of the smallest of metal- and non-metal mine-shaped objects is possible including the most difficult mines to detect: Non-metallic AP mines. Future work will include probe and near field corrections, extensions of signal processing, and image processing. Our system is to be used as a part of a high-efficient multisensor mine-detection system.

\section{References}

[1] K. Iizuka and A. P. Freundorfer, "Detection of Non-metallic Buried Objects by a Step Frequency Radar," Proc. IEEE, vol. 71, Feb. 1983.

[2] Stepped-frequency Ground-Penetrating Radar for Detection of Small Non-metallic Buried Objects, K. B. Jakobsen, H. B. D. Sorensen, and O. Nymann, SPIE Proceedings 'Detection and Remediation Technologies for Mines and Minelike Targets II', vol. 3079, Apr. 1997.

[3] Y. Nagashima, H. Yoshida, J. Masuda, and R. Arioka, "Single-Unit Underground Radar Utilizing Zero-Crossed Synthetic Aperture," IEICE Trans. Communications, vol. E76-B, pp. 1290-1296, Oct. 1993.

[4] S. Koppenjan and M. Bashforth, "Underground and Obscured Object Imaging and Detection," SPIE Proceedings, vol. 1942, Apr. 1993.

[5] M. Kvam, K. K. Mei, and D.J. Angelakos, "Experimental Verification of Computation of Scattering by Flush-Buried Objects, "IEEE Trans. Antennas. Propagation, vol. AP-37, pp. 509-512, Apr. 1989. 


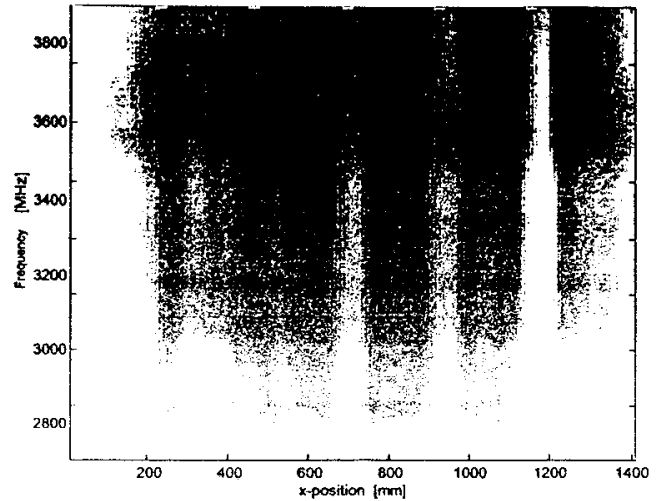

Fig. 2 Amplitude-spectrogram generated from scanning the box containing six metal- and non-metal mine-shaped objects, see Table 1. The positions of the mine-shaped objects with respect to the $x$-direction are illustrated by small white rectangles. The intensities in the figure represent amplitude values in $\mathrm{dB}$. The spectrogram represents the frequency content along one physical scan line, i.e. the $y$-position of the probe is fixed $(y=$ $100 \mathrm{~mm}$ ). The probe step length in the $\mathrm{x}$-direction is $\Delta x=10 \mathrm{~mm}$.

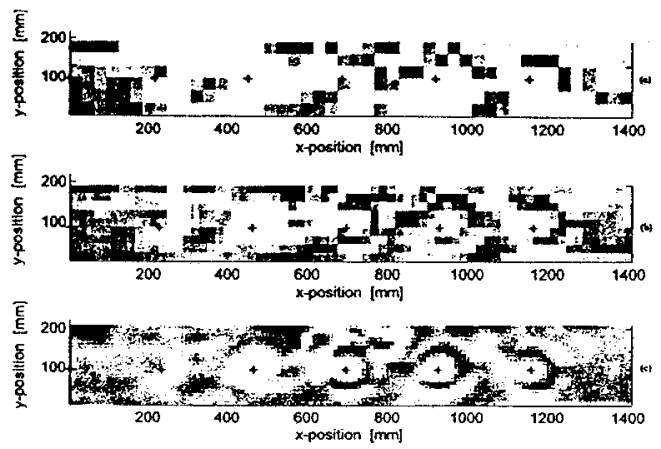

Fig. 4 Radar images, with different resolutions, generated using the phase stepped-frequency radar method. In Fig. 4 (a) the resolutions are $\Delta x$ $=\Delta y=30 \mathrm{~mm}$, in (b) $\Delta x=\Delta y=20 \mathrm{~mm}$, and in (c) $\Delta x=\Delta y=10 \mathrm{~mm}$. Fig. 4 (a), (b) and (c) are thus generated from 7, 10, and 21 collapsed phasespectrograms, respectively. Spectrogram bandwidth is $3,600-3,900 \mathrm{MHz}$ (freq. step size $3 \mathrm{MHz}$ ), i.e. $N=100$. All images are based on a color scale.
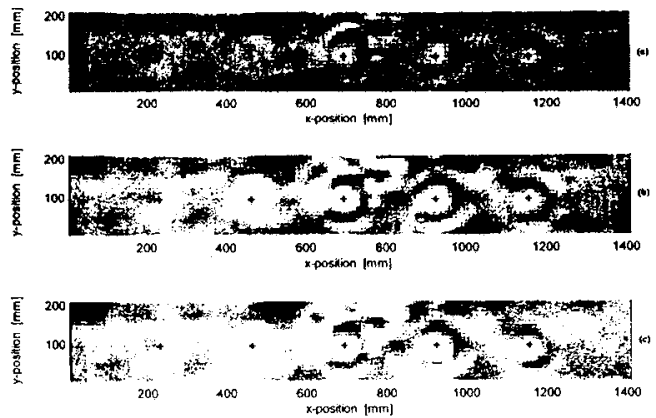

Fig.6 Radar images, generated using: (a) the phase stepped-frequency radar method, (b) the phase stepped-frequency radar method, followed by template crosscorrelation applying a binary mine template $3 \times 3$ matrix, (c) the phase stepped-frequency radar method, followed by template crosscorrelation applying a binary mine template $5 \times 5$ matrix. All images are based on a linear gray scale.

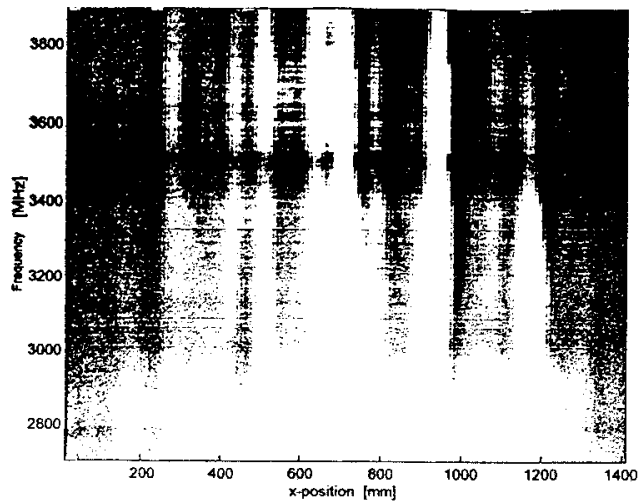

Fig. 3 Phase-spectrogram generated from scanning the box containing six metal- and non-metal mine-shaped objects, see Table 1 . The positions of the mine-shaped objects with respect to the x-direction are illustrated by small white rectangles. The intensities in the figure represent phase values in degrees. The spectrogram represents the frequency content along one physical scan line, i.e. the $y$-position of the probe is fixed $(y=100 \mathrm{~mm})$. The probe step length in the $\mathrm{x}$-direction is $\Delta \mathrm{x}=10 \mathrm{~mm}$.

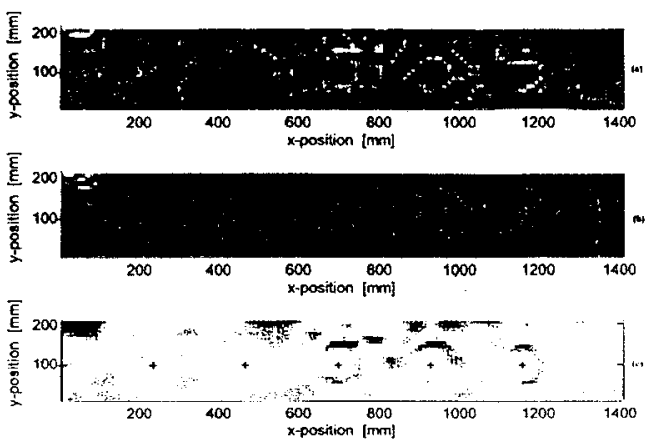

Fig. 5 Radar images, based on different bandwidths, generated using the phase stepped-frequency radar method. The frequency ranges of the phase-spectrograms, applied to generate the three images, are 3,825-3,900 $\mathrm{MHz}(N=25), 3,750-3,900 \mathrm{MHz}(N=50)$, and $3,600-3,900 \mathrm{MHz}(N=$ 100 ) in Fig. 5 (a), (b), and (c), respectively. The frequency step size is 3 $\mathrm{MHz}$ in all cases. All images are based on a color scale.
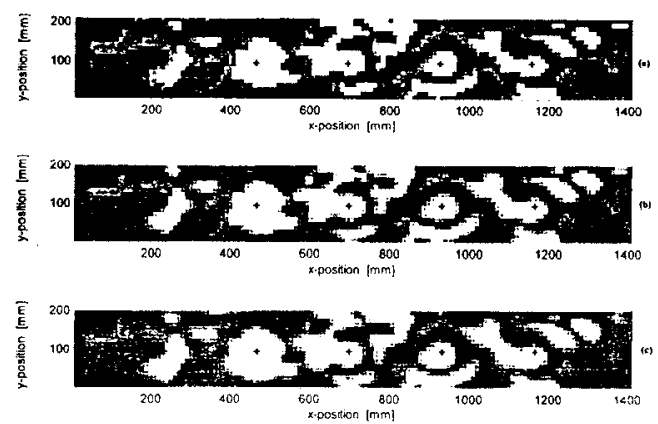

Fig.7 Radar images, generated using: (a) the phase stepped-frequency radar method followed by quarternary image generation, (b) the phase stepped-frequency radar method, followed by quarternary image generation and template crosscorrelation using a binary mine template $3 \times 3$ matrix, (c) the phase stepped-frequency radar method, followed by quarternary image generation and template crosscorrelation applying a binary mine template $5 \times 5$ matrix. All images are based on a linear gray scale. 